\title{
Influence of the Corneal Collagen Cross-linking on Levels of Il- 1b, Il-6, Il-10 and MMP-2 in the Tear film of Patients with Keratoconus.
}

\author{
MD. Karol Krycia ${ }^{1}$, Asst Prof. Dorota Wyględowska-Promieńska ${ }^{1,2}$, \\ MD. Łukasz Kulesza ${ }^{1}$, PhD. Piotr Gościniewicz ${ }^{1}$, PhD. Łukasz Drzyzga ${ }^{1}$, \\ MD. Agata Niedzielska-Krycia ${ }^{1}$, PhD. Tomasz Wilczyński ${ }^{1}$, \\ MD. Krzysztof Maria Wilczyński ${ }^{3}$ \\ ${ }^{1}$. Department of Ophthalmology, Prof. K. Gibiński University Clinical Center of Medical University of Silesia in \\ Katowice, Ceglana 35 street , 40-514 Katowice \\ ${ }^{2}$ Clinic and Department of Ophthalmology, Medical University of Silesia, Ceglana 35 street, 40-514 Katowice. \\ ${ }^{3}$ Students' Scientific Society, Department of Ophthalmology, Medical University of Silesia in Katowice, Poland
}

\begin{abstract}
-
Aim of the study: Evaluation of influence of the corneal collagen cross-linking $(C X L)$ on concentrations of selected cytokines and metalloproteinase 2 in a tear film. Prospective study with a control group.

Methods: 31 eyes of 31 patients, with confirmed KC, were qualified into the study and underwent a collagen cross linking procedure. Samples of tear film $(100 \mu l)$ were collected after 6 weeks from the procedure using spongostan sticks. After centrifugation, the acquired material was tested using high sensitivity ELISA.

Results: In study group before CXL concentrations of IL-1b, IL-6, IL-10, MMP-2 equaled respectively: $151 \pm$ 42, $24 \mathrm{pg} / \mathrm{ml}, 7,44 \pm 1,4 \mathrm{pg} / \mathrm{ml}, 15,88 \pm 2,01 \mathrm{pg} / \mathrm{ml}, 17,49 \pm 2,49 \mathrm{ng} / \mathrm{ml}$. After CXL their levels equaled: 35,32 \pm $13,72 \mathrm{pg} / \mathrm{ml}(p<0,01), 17,73 \pm 5,31 \mathrm{pg} / \mathrm{ml}(p<0,01), 16,17 \pm 2,15 \mathrm{pg} / \mathrm{ml}(p>0,05), 30,71 \pm 3,88 \mathrm{ng} / \mathrm{ml}(p<0,01)$. There was statistically significant correlation between levels of $I L-1 b$ and $M M P-2(r=-0,5 ; p<0,01)$.

Conclusions: Outcomes seem to confirm the hypothesis that inflammation is an essential component of multifactorial etiology of KC. CXL procedure leads to decrease in IL-1b levels and increase in IL-6 and MMP-2 levels in the tear film of patients with $K C$, after six weeks from procedure. There was a statistically significant negative correlation between $\mathrm{Il}-\mathrm{Ib}$ and MMP-2. Evaluation of corneal cytokines may be utilized as the prognostic factor of $K C$ progression.
\end{abstract}

Date of Submission: 28 November $2016 \longrightarrow$ Date of Accepted: 15 February 2017

\section{INTRODUCTION}

Keratoconus (KC) is the most common primary corneal ectasia [1]. It is a stromal dystrophy, classified as a progressive, noninflamatory state characterized by lesions leading to decrease in thickness of central and pericentral cornea and consequent hallmark "cone-shaped" form [2]. KC is characterized by a distortion of the biochemical composition of certain corneal layers: a deficit of collagen type VII, XII and VI in the epithelium and of type I, III, V, VI and XII in the stroma. Progression of a disease leads to the decrease of visual acuity and in advanced stages to the perforation of a cornea. Estimated occurrence rate of KC is around 1/500-1/2000 people, with no gender differences [3]. First symptoms of conus may appear during adolescence, although the most acute phase of disease usually develops around the second to third decade of life. In subsequent years, the disease usually stabilizes, nevertheless the risk of a rapid exacerbation should always be taken into account. Etiology of the keratoconus remains unclear, although due to the development of diagnostic methods, there is a growing number of studies trying to establish reliable hypothesis about its etiopathogenesis. Occurrence of $\mathrm{KC}$ is usually linked to the inter allia eye rubbing, atopy, usage of contact lens, genetic predispositions (Down's syndrome, Ehlers-Danlos' syndrome, Marfan's syndrome) and others. What is more, as the sources in the literature highlights, there is a significant tendency for $\mathrm{KC}$ to coexist with rosacea.

Considering the etiology of keratoconus on a molecular level, particular attention is usually given to the role of proinflammatory cytokines - interleukins, metalloproteinases and growth factors. Interleukins, produced by epithelium and mesothelium of a cornea, belong to the group of growth factors stimulating lymphocytes divisions [4]. Interleukin-1 (Il-1) -occurs in two types: $\alpha$ and $\beta$, where the latter one is responsible for most of the known qualities, inter allia for the modulation of metalloproteinases' and collagenases' activity. Typical for $\mathrm{KC}$ increased activity of IL-1 $\beta$ leads to lesions in keratocytes and the subsequent apoptosis $[5,6,7]$. Il-1 is the 
main factor responsible for induction of IL-6 synthesis, which in turn is an essential part of a sequence of events leading to the chronic inflammation of cornea, the activity of which was found to be increased in course of $\mathrm{KC}$ [4]. An increased level of the abovementioned interleukin was observed in persons liable to frequent microtraumas of the cornea. Antagonistic effects to the Il-1 are exhibited by Il-10, which plays a major role in inhibiting inflammatory and immunological reactions [8]

Metalloproteinases of the extracellular matrix (MMP) belong to the group of zinc-dependent proteolytic enzymes [9]. They are classified as endopeptydases, whose basic function is to participate in the physiological and pathological processes of remodeling and degradation of the e components of extracellular matrix. MMP-2 is a key metalloproteinase in cornea [4], it is believed, that its increased levels are responsible for collagen type IV degradation, and subsequent thinning of corneal tissues, which is a characteristic symptom of keratoconus.

One of the most common and well established methods of therapy in course of a keratoconus, is a collagen cross-linking procedure, with application of a riboflavin activated by an ultraviolet radiation - UVA. Local anesthesia is performed using a proksymetakain hydrochloride solution, and subsequently surgical drapes and lid dilator are applied. Next, a conjunctival sac is flushed with a $10 \%$ solution of a povidon iodine. Afterwards $8 \mathrm{~mm}$ abrasion of a corneal epithelium is conducted, with a special attention to leaving the limbus area intact. In order to minimize the exposition to the UVA radiation reaching the posterior segment of an eye, a $2 \%$ pilocarpine hydrochloride is applied. Further steps include infusing cornea with a 0,1\% riboflavin in $20 \%$ dextran solution, administered every 2 minutes for 30 minutes. Subsequently cornea is being exposed to UVA (wave length $365 \mathrm{~nm}$ and intensity $3 \mathrm{~mW} / \mathrm{cm}^{2}$ ) radiation for the next 30 minutes, from a distance of $40 \mathrm{~mm}$ with a simultaneous administration of riboflavin. After the procedure a 'band-aid' contact lens is applied and anterior segment is examined with a slit lamp in white and red-free light [10]. The connection of riboflavin and UVA radiation leads to the creation of additional bonds between collagen fibers [11] which create covalent bonds in the stroma. This in turn leads to blocking of an irregular astigmatism increase and therefore the decrease in probability of progression of keratoconus [12]. Conduction of a procedure may prevent the necessity of a corneal transplantation in the future.

Aim of our study was to evaluate and analyze variations of MMP-2, IL-1 $\beta$, Il-6, Il-10 levels, before and after CXL procedure in patients with confirmed keratoconus.

\section{MATERIALS AND METHODS}

31 eyes of 31 patients with diagnosed keratoconus, admitted to the Prof. K Gibinski University Medical Center of the Medical University of Silesia, were included into the study and subjected to the collagen crosslinking procedure. During a qualification process, due to the limited amount of female participants, decision was made to limit the study group only to males. Average age in group equaled 27 years (min 17; max 44). In all patients qualified to the control group there were no signs of progression of keratoconus during a whole period of observation. All participants gave an informed consent for participation in the study, and permission from a Bioethical Committee of Medical University of Silesia in Katowice was obtained (permission $n^{0}{ }^{20120217}$ ). Inclusion criteria included: age between 15-45 years, ophthalmological examination consisting of interview, refraction, visual acuity with and without best optical correction, examination of the anterior and posterior segment of an eye in slit lamp, intraocular pressure measured using Goldman's aplanation tonometer and corneal topography performer using Cornea/Anterior segment OCT SS-1000 manufactured by TOMEY, in purpose of clinical confirmation of keratoconus. Qualification of patients into groups was performed based on similarity of a clinical stage of the disease (early (I) and moderate (II) stage according to the Krumeich and Amsler's scale (tab. 1)), topographical (Ks (the steepest meridian) and $\mathrm{Kf}$ (the most flat meridian)) and pachymetric parameters (the minimum pachymetry atop the cone at least $400 \mu \mathrm{m}$ ). Thickness of cornea on the top of the cone was the most important factor conditioning safety during CXL. Exclusion criteria included: age below 15 or above 45, inflammatory diseases precluding from sample collection, state after refraction surgery, scarring of a cornea, state after inflammation of anterior or posterior segment of a vascular membrane, state after ocular trauma, pseudophakia, pregnancy and breastfeeding.

Table 1. Amsler's and Krumeich's classification of keratoconus.

\begin{tabular}{|c|c|c|c|c|c|}
\hline \multicolumn{6}{|c|}{ Amsler's and Krumeich's classification of keratoconus } \\
\hline Stage & $\begin{array}{lr}\begin{array}{l}\text { Refractive } \\
\text { (myopia }\end{array} & \begin{array}{r}\text { error } \\
\text { and/or }\end{array} \\
\text { astigmatism) } & \\
\end{array}$ & $\begin{array}{l}\text { Corneal curvature } \\
\text { radius } \\
\text { central value } \mathrm{K} \text { ) }\end{array}$ & Notes & & $\begin{array}{l}\text { Minimal cornea } \\
\text { thickness (pachymetry) }\end{array}$ \\
\hline I & $<5 \mathrm{D}$ & $<48 \mathrm{D}$ & $\begin{array}{lc}\text { Off-axis } & \text { cornea } \\
\text { Vogt } & \text { lines } \\
\text { no cornea scarring } & \end{array}$ & $\begin{array}{l}\text { convex, } \\
\text { present, }\end{array}$ & $>460 \mu \mathrm{m}$ \\
\hline II & $5-8 \mathrm{D}$ & $48-53 \mathrm{D}$ & $\begin{array}{l}\text { Vogt lines } \\
\text { no cornea scarring }\end{array}$ & present, & $400-460 \mu \mathrm{m}$ \\
\hline III & 8-10 D & $53-55 \mathrm{D}$ & Vogt lines & present, & $200-400 \mu \mathrm{m}$ \\
\hline
\end{tabular}


Influence of the Corneal Collagen Cross-linking on Levels of Il-1b, Il-6, Il-10 and MMP-2 in the .....

\begin{tabular}{|l|l|l|l|l|}
\hline & & no cornea scarring & \\
\hline IV & $\begin{array}{l}\text { Unable to measure } \\
\text { refractive terror }\end{array}$ & $>55 \mathrm{D}$ & $\begin{array}{l}\text { Sparring in the central part of the cornea, } \\
\text { Perforation of the cornea }\end{array}$ & $<200 \mu \mathrm{m}$ \\
\hline
\end{tabular}

Samples of a tear film were collected twice. Tear liquid volume of at least $100 \mu 1$, was acquired by placing 3 spongostan sticks in a conjunctival sac for a period of 2 minutes. First sample was collected directly before CXL, and second one 6 weeks after procedure. Upon removal of spongostan sticks, $10 \%$ Sulfacetamidum Natricium antibiotic drops were administered to the conjunctival sac as an anti-inflammatory prophylaxis. Acquired material was separated directly after collection by centrifugation lasting 5 minutes, with a speed of 10000 rotations per minute, using a Centrifuge MPW-300 (MPW Med. Instruments Poland) device. Subsequently elicted biological material was stored in $-20^{\circ} \mathrm{C}$. After administration of a local anesthesia, using a proksymetakain hydrochloride solution, and following application of surgical drapes and lid dilator, the conjunctival sac was flushed with a $10 \%$ solution of a povidon iodine. Afterwards an $8 \mathrm{~mm}$ abrasion of a corneal epithelium was conducted, with a special attention to leaving the limbus area intact. In order to minimize the exposition to the UVA radiation reaching the posterior segment of an eye, a $2 \%$ pilocarpine hydrochloride was applied. Further steps included infusion of cornea with a $0,1 \%$ riboflavin in $20 \%$ dextran solution, administered every 2 minutes for 30 minutes. Subsequently cornea was being exposed to UVA (wave length $365 \mathrm{~nm}$ and intensity $3 \mathrm{~mW} / \mathrm{cm}^{2}$ ) radiation for the next 30 minutes, from a distance of $40 \mathrm{~cm}$ with a simultaneous administration of riboflavin. After the procedure a 'band-aid' contact lens was applied and anterior segment was examined with a slit lamp in white and red-free light [10].Postoperative treatment was applied to all participants undergoing CXL procedure, including application of "band-aid" contact lens (Alcon) for a period of 1 week and administration of an antibiotic prophylaxis (Levofloxacinum $5 \mathrm{mg} / \mathrm{ml}$ manufactured by Santen OY) 4 times a day. One week after procedure, "band-aid" contact lens were removed, and Dexamethason $1 \mathrm{mg} / \mathrm{ml}$ (Laboratories Thea) drops were applied for a period of 4 weeks, with administration frequency of 3 times a day, decreasing by one each week. Moisturizing drops Cationorm (Santen OY) and regenerating ointment Corneregel $50 \mathrm{mg} / \mathrm{ml}$ (Dr. Gerhard Mann Chem-Pharm. Fabrik VP Valeant) were applied for the whole postoperative period. 6 weeks after collection of the first sample, complete ophthalmological examination was conducted, including the same procedures as during inclusion into study. Each $100 \mu \mathrm{l}$ sample of a tear liquid was divided into $20 \mu \mathrm{l}$ portions, and diluted with $100 \mu \mathrm{l}$ of a solvent included into the test kit, in order to conduct evaluation of levels of IL-1 $\beta$, Il-6, Il-10 and MMP-2 contained in a sample. The dilution factor was taken into account during a statistical analysis. All biochemical procedures were conducted using a Diaclone $450 \mathrm{~nm}(0,1 \mathrm{~s})$ quantitive reader to a high-sensitivity ELISA and Kit Human tests.

Statistical analysis was conducted using commercially available software GraphPad Prism 6. Accepted level of significance equaled $\alpha=0,05$. Data was prepared for further analysis using a Kolmogorov-Smirnov's test and D'Agostino\& Pearson omnibus test. Standard deviation and average was calculated. In order to compare outcomes between groups, Wilcoxon signed rank test, Mann-Whitney's U test and Dunn's multiple comparison tests were applied.

\section{OUTCOMES}

31 patients with diagnosed keratoconus participated in the study (tab. 2). Il-1 $\beta$, IL-6, IL-10 and MMP-2 levels in material obtained from a conjunctival sac were analyzed using high sensitivity ELISA, and outcomes are presented in a table number 3 .

In all patients, Il-1 $\beta$ level was significantly decreased after 6 weeks period of observation, in comparison to values obtained before CXL ( $<<0,01)$ [pic. 1]. Inversely in case of Il-6 a statistically significant raise in concentrations was observed in a follow-up period $(\mathrm{p}<0,05)$ [pic. 2]. There were no statistically significant differences in concentrations of Il-10 [pic. 3]. Analysis of MMP-2 concentrations in tear film revealed major, statistically significant increase in patients after CXL in comparison to samples acquired before procedure [pic. 4]. In course of a statistical analysis, a significant negative correlation has been found between concentrations of Il-1 $\beta$ and MMP-2 in a tear film acquired from patients who underwent the CXL procedure $(\mathrm{r}=-$ $0,5 ; \mathrm{p}<0,01)$ [pic. 5]. There was no statistically significant correlation between increase in IL-6 concentrations and MMP-2 in patients in follow-up period.

\section{DISCUSSION}

Selection of cytokines included into a biochemical analysis was based on their properties. Il-1 $\beta$ and Il-6 were selected due to the strongest proinflammatory qualities, and Il-10 due to its anti-inflammatory properties. Data available in literature highlights a significance of MMP-9 in corneal remodeling. MMP-2, belonging to the same group of gelatinases, was included into analysis.

Despite plenty of publications describing role of inflammatory mediators in a pathogenesis of the keratoconus, data about influence of particular cytokines on a progression of $\mathrm{KC}$ is still limited. Acquired data 
revealed that CXL significantly decreases concentrations of Il-1 $\beta$, increases levels of II-6 and MMP-2, whereas does not affect Il-10 concentrations. Elevated concentrations of Il-6 may be related to the presence of a significant local inflammatory reaction, what was revealed by Gavis[2], Shetty[13], Jun[14], Lema [15,16] in their studies, which compared its levels in healthy subjects and patients with the keratoconus. This hypothesis was also supported by Sarkhabi et al [17]. Aforementioned considerations were widened by Wojcik et al. [4] revealing further increase of Il-6 concentration during an exacerbation of keratoconus. Significant data was also provided by Becker et al.[18] who observed a considerable elevation of Il-6 level in ulcerated corneas in comparison to corneas with scarring or dystrophy. Other hypothesis trying to explain etiology of the keratoconus, states that it may be caused by microtraumas resulting from for example forceful eye rubbing or wearing contact lenses. Mechanical damage caused by microtraumas may affect Il-6 levels, increasing the risk of development of keratoconus. Balasubramanian et al. [19] revealed a significant elevation in Il-6 concentrations after 60 seconds of eye rubbing by healthy volunteers. Confirmation of an aforementioned hypothesis may also be found in outcomes of a study conducted by Wilson et al. [20] who revealed 2-40 times higher concentrations of proinflammatory cytokines, such as Il-6, in people using contact lenses. Among surgical procedure aiming to slow down or stop the progression of keratoconus, CXL is considered to be a golden standard. Therefore it was of the interest how it affects Il- 6 concentrations. Kolozsvari et al. [1] evaluated influence of CXL on levels of cytokines in tear film of patients with KC, to date it was first and only publication on this issue. They revealed statistically significant increase in Il-6 level on a fourth day after intervention, and decrease in its concentration after six months of observation. Kolozsvari et al. allotted an annual observation period in their study. In reference to our results it can be stated that decrease in Il-6 concentration occurs at least six weeks after the procedure. Postoperative pharmacotherapy in aforementioned study differed from a treatment administered in our center. Patients in our study used steroidal drops over the period of 4 weeks, while participants of Kolozsvari's project for 3 months. Noted was a statistically significant increase of he analyzed mediator that persisted 6 weeks after the CXL procedure.

Elevated levels of II-1 in patients with an inflammation of the cornea were first noticed by Becker et al. [21], who compared its concentrations in tear film of patients with an inflammation and with scarring of the cornea. Sarkhabi et al. [17] revealed increased levels of Il-1 $\beta$ in tear film of patients with diagnosed keratoconus. In our study, obtained concentrations of Il-1 $\beta$ were considerably higher than Sarkhabi's, and its level diminished after CXL procedure. This observation implies that Il-1 is an essential factor in pathogenesis of $\mathrm{KC}$, and supports inflammatory hypothesis. Moreover, data obtained in our study reveals that the more advanced stage of keratoconus is, the higher Il-1 concentration in the tear film is. Significance of microtraumas in pathogenesis of KC may be confirmed by an elevation of Il-1 concentrations in the tear film of patients with mechanical damage to the corneal epithelium [20]. Keratocytes in patients with $\mathrm{KC}$ have four times higher amount of Il-1 receptors [22]. In the same time Il-1 is a modulator of keratocyte proliferation, differentiation and apoptosis. Its increased emission was observed during microtraumas of the epithelium. In patients with rosacea, an elevated expression of genes from Il-1 family was confirmed. Due to that, widely brought up in a literature issue, of the coexistence of the rosacea and the keratoconus, seems to be especially interesting. Currently numerous studies, aiming at description of a polymorphism for Il-1 $\beta$ gene present in patients with KC, are being conducted worldwide [23, 24, 25,26,27]. Results obtained in our study reveal decrease in IL-1 concentrations after CXL, what may stand for the efficacy of the CXL procedure in keratoconus treatment.

Mascai et al. [3] and Lema et al. [18] in an attempt to confirm the participation of the corneal microtrauma in pathogenesis of $\mathrm{KC}$, examined the tear film cytokines concentrations of people using contact lenses. Data obtained by them, reveals 8 times higher level of anti-inflammatory interleukin 10. Sorkhabi et al. [17] focusing on comparisons of the tear film composition in patients with $\mathrm{KC}$ and healthy controls, found out a decreased concentration of Il-10 in patients. Data obtained in our study, in patients with $\mathrm{KC}$ who underwent CXL procedure and in control group, revealed no statistically significant difference. Levels of Il-10 requires further research, focusing on their shifts and participation in pathogenesis of $\mathrm{KC}$ and other inflammatory diseases of anterior segment of an eye.

Balasubramanian et al.[29] revealed increase in MMP-1, -3, -7, -13 in the tear film of patients with keratoconus. Similar results were publish by inter allia Jun [14] and Lema $[16,18]$. In their study, Wójcik et al. [4] point out that increase in MMP activity, observed in course of KC, probably leads to the degradation of an extracellular matrix leading to thinning of corneal tissues. Elevated levels of MMP-2 may be explained by an apoptosis of keratocytes and appearance of additional bonds between collagen fibers, aiming to stabilize spherical shape of cornea. In a following study, we observed increased levels of MMP-2 in the tear film of patients with KC six weeks after the CXL procedure. Taking into account the fact that MMP-2 belongs to the same family as MMP-9, and exhibit similar properties and functions, outcomes obtained by Shetty et al. [13] seem to be relevant in reference to our results. In their study, Shetty et al. observed patients with KC over the period of 6 months, during pharmacotherapy with a Cyclosporine A. Data obtained in course of their research, 
revealed decrease in MMP-9 levels in the tear film of patients, and subsequent stabilization of a clinical condition. Therefore the administration of Cyclosporine A directly after CXL procedure seems to be inadvisable as it may disturb the remodeling and hardening of the stroma of the cornea. In the future, the evaluation of MMP-2 concentrations may be utilized as a prognostic factor for assessment of CXL outcomes.

\section{CONCLUSIONS}

Following study confirms inflammatory hypothesis in multifactorial pathogenesis of KC. CXL procedure leads to the decrease of IL-1 $\beta$ concentrations, increase in Il-6 and MMP-2 levels and does not affect Il-10 level. There was a statistically significant negative correlation between Il-1 $\beta$ and MMP-2. Subsequently to the conducted CXL procedure, inflammatory reaction subsided and concentrations of MMP-2, responsible for remodeling of a cornea, increased. In our opinion evaluation of Il-1 $\beta$ concentrations, as a main proinflammatory cytokine affecting the corneal pathology, should be continued. Assessment of proinflammatory cytokines may be utilized as a prognostic factor of a progression of keratoconus.

Table. 2 Patients which participated in the study

\begin{tabular}{|l|l|l|l|l|}
\hline $\mathrm{N}$ & Age & Maximum age & Minimum age & Males \\
\hline 31 & 27 & 64 & 17 & $31(100 \%)$ \\
\hline
\end{tabular}

\begin{tabular}{|c|c|c|}
\hline & Study & Stydy after 6 weeks \\
\hline \multicolumn{3}{|l|}{ IL-1beta } \\
\hline Mean [pg/ml] & 151 & 35,32 \\
\hline Std. Deviation & 235,2 & 76,38 \\
\hline Std. Error & 42,24 & 13,72 \\
\hline KS distance & 0,2711 & 0,3301 \\
\hline $\mathrm{P}$ value & $<0,0001$ & $<0,0001$ \\
\hline \multicolumn{3}{|l|}{ IL -6 } \\
\hline Mean [pg/ml] & 7,439 & 17,73 \\
\hline Std. Deviation & 7,878 & 29,59 \\
\hline Std. Error & 1,415 & 5,314 \\
\hline KS distance & 0,1725 & 0,2745 \\
\hline $\mathrm{P}$ value & 0,0194 & $<0,0001$ \\
\hline \multicolumn{3}{|l|}{ IL-10 } \\
\hline Mean [pg/ml] & 15,88 & 16,17 \\
\hline Std. Deviation & 11,2 & 11,97 \\
\hline Std. Error & 2,011 & 2,15 \\
\hline KS distance & 0,1313 & 0,2022 \\
\hline $\mathrm{P}$ value & $>0,10$ & 0,0023 \\
\hline \multicolumn{3}{|l|}{ MMP-2 } \\
\hline Mean [ng/ml] & 17,49 & 30,71 \\
\hline Std. Deviation & 13,64 & 21,26 \\
\hline Std. Error of Mean & 2,491 & 3,882 \\
\hline KS distance & 0,1717 & 0,1253 \\
\hline $\mathrm{P}$ value & 0,0243 & $>0,1000$ \\
\hline
\end{tabular}

Table 3. Obtained levels of cytokines and MMP-2.

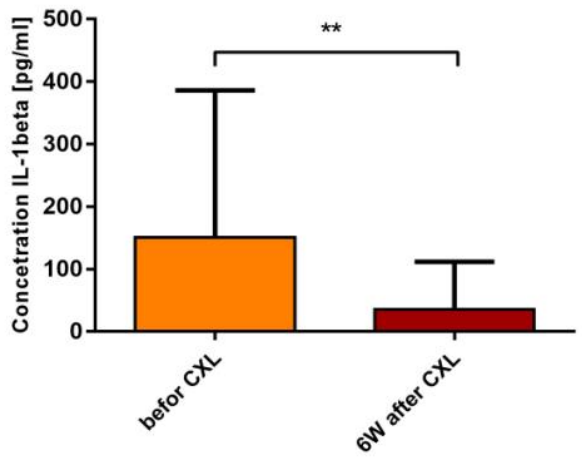

Pic.1 Difference in concentrations of IL-1 $\beta$ before and after CXL procedure. 


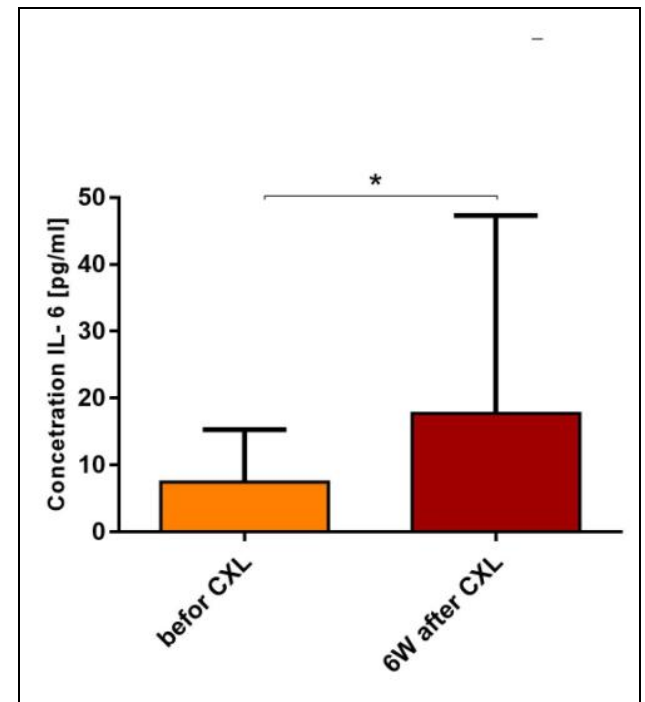

Pic.2 Difference in concentrations of IL-6 before and after CXL procedure.

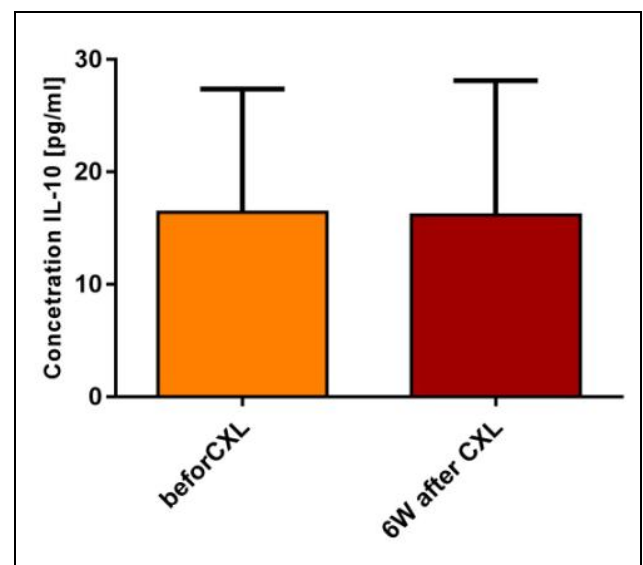

Pic 3. Difference in concentrations of IL-10 before and after CXL procedure.

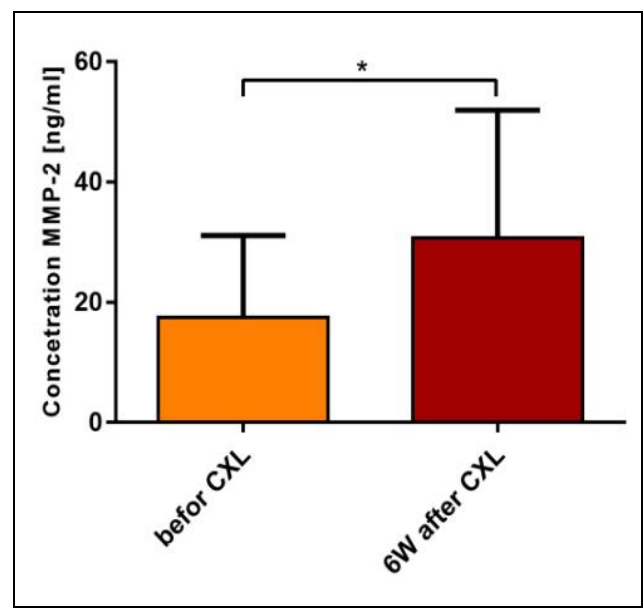

Pic 4. Difference in concentrations of MMP-2 before and after CXL procedure. 


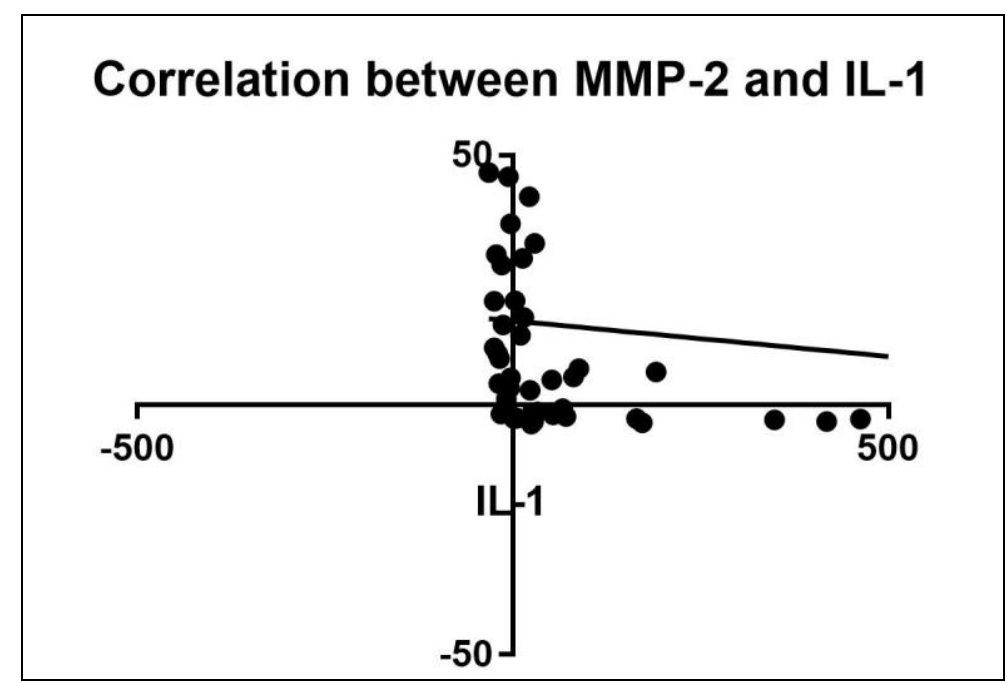

Pic 5. Correlation between concentrations of MMP-2 and Il-1.

\section{BIBLIOGRAPHY}

[1]. Kolozsvári BL, Berta A, Petrovski G, Miháltz K, Gogolák P, Rajnavölgyi E, Hassan Z, Széles P, Fodor M.:”Alterations of tear mediators in patients with keratoconus after corneal crosslinking associate with corneal changes.”;PLoS One. 2013;8(10):e76333. doi: 10.1371/journal.pone.0076333. eCollection 2013.

[2]. Galvis V1, Sherwin T2, Tello A1, Merayo J3, Barrera R4, Acera A5.: "Keratoconus: an inflammatory disorder?"; Eye (Lond). 2015; 29(7):843-59. doi: 10.1038/eye.2015.63. Epub 2015 May 1.

[3]. Macsai MS, Varley GA, Krachmer JH.:"Development of keratoconus after contact lenswear: Patient characteristics."; Arch Ophthalmol 1990;108:534-8.

[4]. Wojcik KA, Blasiak J , Szaflik J , Szaflik JP:"Faculty of Biology and Environmental Sciences, Department of Molecular Genetics, University of Lodz, Łódź, Poland.”; Acta Biochimica Polonica 2014; 61(1):55-62

[5]. Wilson SE, He YG, Lloyd SA: "EGF, EGF receptor, basic FGF, TGF beta-1, and interleukin-1alpha mRNA in human corneal epithelial cells and stromal fibroblasts."; Invest Ophthalmol Vis. Sci 2012; 33:1756-1765.

[6]. Wilson SE, He YG, Weng J, Li Q, McDowall AW, Vital M, Chwang EL: "Epithelial injury induces keratocyte apoptosis: hypothesized role for the interleukin-1 system in the modulation of corneal tissue organization and wound healing."; Exp Eye Res 1996; 62: 325-327.

[7]. Wise R, Wilson SE, He YG, Weng J: "Interleukin-1 alpha induces apoptosis in human corneal stromal fibroblast cells."; Invest Ophthalmol Vis Sci 1994; 35: 1980.

[8]. Grunauer-Kloevekorn C, Duncker G.I: Keratoconus: "Epidemiology, risk factors and diagnosis.”; Klin Monl Augenheilkd. 2006; 223(6):493-502

[9]. Wasiliewska A.:"Role of matrix metalloproteinases (MMP) and their tissue inhibitors (TIMP) in nephrology.”; Przegląd Lekarski 2009; 66(9)

[10]. Kucharzewski P.: „Analysis of the morphology of the cornea using confocal microscopy in vivo in patients with keratoconus after surgery crosslinking of collagen fibers"; SUM 2012: p34

[11]. Dahl B, Spotts E, Truong J:“Corneal collagen cross-linking: An introduction and literature review.”; Optometry 2012; 83: 33-42.

[12]. Mirko R, Jankov II, Jovanovic V, Nikolic L, . Lake JC, Kymionis G, Coskunseven E.: “Corneal Collagen Cross-Linking.”; Middle East Afr J Ophthalmol. 2010; 17(1)

[13]. Shetty R, Ghosh A, Lim RR, Subramani M, Mihir K, Reshma AR, Ranganath A, Nagaraj S, Nuijts RM, Beuerman R, Shetty R, Das D, Chaurasia SS, Sinha-Roy A, Ghosh A.: "Elevated expression of matrix metalloproteinase-9 and inflammatory cytokines in keratoconus patients is inhibited by cyclosporine";Invest Ophthalmol Vis Sci. 2015:56(2):738-50. doi: 10.1167/iovs.14-14831.

[14]. Jun AS, Cope L, Speck C, Feng X, Lee S, Meng H, Hamad A, Chakravarti S.: "Subnormal cytokine profile in the tear fluid of keratoconus patients.”;PLoS One.2011;6(1):e16437. doi: 10.1371/journal.pone.0016437.

[15]. Lema I, Sobrino T, Durán JA, Brea D, Díez-Feijoo E.: "Subclinical keratoconus and inflammatory molecules from tears."; Br J Ophthalmol. 200;93(6):820-4. doi: 10.1136/bjo.2008.144253

[16]. Lema I, Durán JA.: "Inflammatory molecules in the tears of patients with keratoconus."; Ophthalmology. 2005;112(4):654-9.

[17]. Sorkhabi R, Ghorbanihaghjo A, Taheri N, Ahoor MH: "Tear film inflammatory mediators in patients with keratoconus."; Int Ophthalmol. 201;35(4):467-72. doi: 10.1007/s10792-014-9971-3

[18]. Lema I, Durán JA, Ruiz C, Díez-Feijoo E, Acera A, Merayo J.:"Inflammatory response to contact lenses in patients with keratoconus compared with myopic subjects. Cornea 2008;27:758-63.

[19]. Balasubramanian SA, Pye DC, Willcox MD.: "Effects of eye rubbing on the levels of protease, protease activity and cytokines in tears: relevance in keratoconus.”; Clin Exp Optom. 2013;96(2):214-8. doi: 10.1111/cxo.12038.

[20]. Wilson SE, He YG, Weng J, Li Q, McDowall AW, Vital M, et al.."Epithelial injury induces keratocyte apoptosis: hypothesized role for the interleukin-1 system in the modulation of corneal tissue organization and wound healing."; Exp Eye Res 1996;62:325-37.

[21]. Becker J., Salla S., Dohmen U., Redbrake C., Reim M. Explorative study of interleukin levels in human cornea. Graefes Arch Clin Exp Ophthalmol 1995; 233(12):766-771

[22]. Bereau J, Fabre EJ, Hecquet C, Pouliquen Y, Lorans G.:"Modification of prostaglandin E2 and collagen synthesis in keratoconus fibroblasts associated with an increase of interleukin-1 alpha receptor number.”; CR Acad Sci III 1993;316:425-30.

[23]. Wang Y, Wei W, Zhang C, Zhang X, Liu M, Zhu X, Xu K.: "Association of Interleukin-1 Gene Single Nucleotide Polymorphisms with Keratoconus in Chinese Han Population.”; Curr Eye Res. 2015; 22:1-6.

[24]. Mikami T1, Meguro A, Teshigawara T, Takeuchi M, Uemoto R, Kawagoe T, Nomura E, Asukata Y, et. al: "Interleukin 1 beta promoter polymorphism is associated with keratoconus in a Japanese population.”; Mol Vis. 2013; 19:845-51 
[25]. Wygledowska-Promieńska D: “Present views concerning surgery treatment of keratoconus.”; Klin Oczna. 2005;107(7-9):525- 8.

[26]. Wygledowska-Promieńska D, Zawojska I.: "Procedure for keratoconus detection according to the Rabinowitz-Rasheed method-personal experience."; Klin Oczna. 2000;102(4):241-4.

[27]. Wygledowska-Promieńska D, Zawojska I.: "Use of AutoTopograf TMS-3 keratoconus screening program for keratoconus detection.”; Klin Oczna. 2000;102(4):237-40.

[28]. Balasubramanian SA, Mohan S, Pye DC, Willcox MD.: "Proteases, proteolysis and inflammatory molecules in the tears of people with keratoconus.”; Acta Ophthalmol. 2012;90(4):e303-9. doi: 10.1111/j.1755-3768.2011.02369.x.

Pic 1. Difference in concentrations of IL-1ß before and after CXL procedure.

Pic 2. Difference in concentrations of IL-6 before and after CXL procedure.

Pic 3. Difference in concentrations of $I L-10$ before and after $C X L$ procedure.

Pic 4. Difference in concentrations of MMP-2 before and after CXL procedure.

Pic 5. Correlation between concentrations of MMP-2 and Il-1.

Table 1. Table 1 Amsler's and Krumeich's classification of keratoconus.

Table 2. Patients which participated in the study.

Table 3 Obtained levels of cytokines and MMP-2. 